\begin{tabular}{|c|c|c|c|}
\hline \multirow{3}{*}{$\begin{array}{r}\text { Case Reports in } \\
\text { Gastroenterology }\end{array}$} & Case Rep Gastroenterol 2017; & & \multirow[b]{2}{*}{$\begin{array}{l}\text { Karger } \\
\text { Opengaccess }\end{array}$} \\
\hline & $\begin{array}{l}\text { DOI: } 10.1159 / 000480071 \\
\text { Publisned onlIne: September 26, } 2017\end{array}$ & $\begin{array}{l}\text { The Author(s) } \\
\text { Published by S. Karger AG, Basel } \\
\text { www.karger.com/crg }\end{array}$ & \\
\hline & $\begin{array}{l}\text { This article is licensed under the C } \\
\text { International License (CC BY-NC) (h } \\
\text { Usage and distribution for commercial }\end{array}$ & $\begin{array}{l}\text { mons Attribution-NonCommercial } 4 . C \\
\text { ger.com/Services/OpenAccessLicense) } \\
\text { quires written permission. }\end{array}$ & \\
\hline
\end{tabular}

\title{
A Patient with Nafcillin-Associated Drug-Induced Liver Failure
}

\author{
Qin Rao Isaiah Schuster Talal Seoud Kevin Zarrabi \\ Nirvani Goolsarran \\ Department of Medicine, Stony Brook University Hospital, Stony Brook, NY, USA
}

\section{Keywords}

Nafcillin · Liver · Jaundice · Drug-induced liver injury

\begin{abstract}
Nafcillin-induced acute liver injury is a rare and potentially fatal complication that has been known since the 1960s but inadequately studied. At this time, the only proven treatment is early discontinuation of the drug. Because of the high prevalence of nafcillin class antibiotic use in the United States, it is important for clinicians to have a high clinical suspicion for this diagnosis. We present a case of liver failure attributable to nafcillin use in a 68-year-old male with a history methicillin-sensitive Staphylococcus and L3/L4 osteomyelitis. After starting long-term antibiotic therapy, he presented with painless jaundice which necessitated discontinuation of the drug. At the time of presentation, the patient's lab work exhibited a bilirubin/direct bilirubin of $9.4 / 8.2 \mathrm{mg} / \mathrm{dL}$, alkaline phosphatase of $311 \mathrm{IU} / \mathrm{L}$, and aspartate transaminase/alanine transaminase of 109/127 IU/L. The patient was switched to i.v. vancomycin given the concern for drug-induced liver injury. Imaging did not show obstruction of the hepatobiliary or pancreaticobiliary trees. Serology was unremarkable for viral etiology, autoimmune processes, Wilson disease, and hemochromatosis. A liver biopsy showed findings consistent with drug-induced liver injury. The patient's liver function tests peaked at day 7 of admission and trended towards normal levels with cessation of nafcillin therapy. The patient was discharged with a diagnosis of nafcillin-induced acute liver injury. Our case highlights the importance of early recognition of the diagnosis and careful monitoring of liver function when nafcillin is employed in the clinical setting.




\section{Introduction}

Cholestatic hepatitis is a rare complication of nafcillin and its class equivalent narrowspectrum beta-lactam antibiotics [1]. A review of the literature reveals that the first case of oxacillin-induced hepatitis was reported in 1965 . Since then, there have been only a few case reports and 2 cohort studies on the subject. The first cohort study by Viehman et al. [2] followed 224 patients at a single institution who were receiving antibiotics for more than 1 month. Within this population, $3 \%$ of oxacillin users and $4 \%$ of nafcillin users developed acute hepatic toxicity, without a significant difference in rates between the 2 groups [2]. The second cohort study followed 222 children on antibiotics for 4 years and revealed an acute hepatitis rate of $22 \%$ for oxacillin and $0 \%$ for nafcillin [1].

It remains unclear if there is a mortality rate associated with nafcillin drug-induced hepatitis. However, both acute drug-induced liver injury (DILI) and its progressive disease, drug-induced liver injury-acute liver failure (DILI-ALF) carry a high morbidity and mortality rate. These disease processes can lead to encephalopathy, hypercoagulability, and acute spontaneous bacterial peritonitis; require ICU level of care, and the need for liver transplantation [3].

Treatment options for DILI/DILI-ALF are limited and the most efficacious approach is withdrawal of the offending agent. Thus, early recognition of the signs of beta-lactaminduced acute hepatic toxicity is imperative to limit the risk of organ failure. Below, we report a recent case of nafcillin-induced hepatotoxicity and provide further insight into this rare complication.

\section{Case Report}

We report a case of a 68-year-old Caucasian man (90 kg, $177.8 \mathrm{~cm}$, BMI 28.47) with a past medical history of hypertension and diabetes mellitus, who presented with new-onset painless jaundice. The patient had recently been hospitalized for treatment of L3/L4 osteomyelitis secondary to methicillin-sensitive Staphylococcus aureus bacteremia. He was treated with intravenous nafcillin, 2 g every $4 \mathrm{~h}$, as an inpatient and was continuing treatment as an outpatient. The patient had been on antibiotic therapy for 4 weeks at the time of presentation. The patient reported that his skin had turned yellow, his urine had grown darker, and his stools had turned grey over a course of 5 days. He also suffered a 20 -lb weight loss over a 3-week period prior to presentation. The patient denied abdominal pain, fever/chills, nausea, vomiting, diarrhea, and sick contacts. The patient further denied any use of alcohol, illicit drugs, herbal supplements, or any new medications except nafcillin.

On presentation, the patient was afebrile and hemodynamically stable. Physical exam revealed intact mentation, scleral and sublingual icterus, trace asterixis, jaundiced skin tone, normal bowel sounds, no abdominal tenderness, and no hepatomegaly. Lab work revealed total bilirubin/direct bilirubin $9.4 / 8.2 \mathrm{mg} / \mathrm{dL}$; alkaline phosphatase of $311 \mathrm{IU} / \mathrm{L}$; aspartate transaminase/alanine transaminase 109/127 IU/L; INR 1.6; ammonia level $17 \mu \mathrm{mol} / \mathrm{L}$; and acetaminophen level $<5 \mu \mathrm{g} / \mathrm{mL}$. The Model for End-Stage Liver Disease (MELD) score was 22 , with $19.6 \%$ estimated 3-month mortality. Eosinophils were elevated to $20.7 \%$ of blood leukocytes.

CT scan of the abdomen and pelvis at the time of initiation of nafcillin therapy on the previous admission revealed a normal-sized liver, an unremarkable gallbladder, no biliary ductal dilation, and no pancreatic lesions. On this admission, a right upper quadrant ultra- 
sound did not identify stones, sludge, or biliary pathology. Further imaging study with an MRCP showed an unremarkable pancreatobiliary tree, no focal lesions in the liver, and no intrahepatic/extrahepatic biliary dilatation.

Hepatic workup included viral hepatitis serology (genotype A, B, and C), antimitochondrial antibody, anti-smooth muscle antibody, total immunoglobulin levels, anti-LK microsomal antibodies, iron studies, and ceruloplasmin levels. All serology and titers were unremarkable (online suppl. Table 1, see www.karger.com/doi/10.1159/000480071). Lactulose was started as a preventive measure against encephalopathy and empiric intravenous $\mathrm{N}$-acetylcysteine was administered. Nafcillin was discontinued on admission and the patient was transitioned to intravenous vancomycin for further treatment of the osteomyelitis given the concern for DILI-ALF.

The patient underwent a percutaneous liver biopsy. Pathology revealed findings consistent with cholestatic hepatitis. Liver enzymes trended upwards and peaked at AST/ALT 313/190 IU/L on day 7 of admission (Fig. 1). Total/direct bilirubin peaked at 13.1/11.6 $\mathrm{mg} / \mathrm{dL}$ on day 4 of admission (Fig. 2). A diagnosis of nafcillin-induced acute liver injury was made. The patient was discharged 10 days after admission with outpatient gastroenterology follow up for further evaluation. Transaminases, bilirubin levels, and the INR decreased to baseline levels with complete resolution of the jaundice at the time of follow up 2 weeks after discharge. The MELD score improved to 17 , with $6.0 \%$ estimated 3-month mortality.

\section{Discussion}

Nafcillin, a semi-synthetic penicillin, is frequently used in the management of disease states caused by gram-positive species. Since the 1960s, the penicillin class of antibiotics has become associated with a number of hepatic and renal toxicities which in many patients may contribute to a high degree of morbidity and mortality [4,5]. The hepatotoxicity is frequently associated with underlying cholestasis that in some cases may present with jaundice and on laboratory evaluation with a significant transaminitis and/or hyperbilirubinemia [6]. At this time, no data have demonstrated a sensitive marker for predicting the onset of this form of injury in the setting of antibiotic use.

This case highlights the importance of early recognition of nafcillin-induced acute liver injury. As demonstrated in this patient, complete withdrawal of nafcillin resulted in complete recovery of liver function and the drug effects were proven reversible and we add to the growing body of literature supporting the finding of DILI. The current opinion is that nafcillin is generally well tolerated, with the most common side effects being mild to moderate gastrointestinal and hematological toxicities. For this reason, treatment discontinuation due to toxicity is a rare occurrence.

A thorough workup of the patient's liver failure was conducted and no underlying etiology was identified to explain the liver failure. The patient had no prior hepatic or renal disease to explain possible decreased clearance of the drug. Moreover, there were no hepatotoxic drugs administered at or around the time of the observed liver failure. Due to the acute nature of the patient's presentation, the cholestatic pattern of the liver injury, and eosinophilia, there was high suspicion for DILI. Pathology further confirmed our diagnosis. Following discontinuation of the medication, the patient's symptomatology dramatically improved and his liver function normalized. Due to the severity of the morbidity/mortality associated with DILI, we believe that patients with long-term treatment with nafcillin and its class 
equivalent narrow-spectrum beta-lactam antibiotics will benefit from serial liver function testing throughout therapy.

In conclusion, we highlight the importance of recognizing drug-induced liver failure secondary to prolonged use of nafcillin. The patient presented with painless jaundice and lab work revealed a marked transaminitis and hyperbilirubinemia. This case highlights the importance of monitoring liver function in patients undergoing nafcillin therapy. If hepatic injury is observed, prompt discontinuation of the therapy should be considered.

\section{Statement of Ethics}

The authors have no ethical conflicts to disclose.

\section{Disclosure Statement}

The authors declare that there is no conflict of interest regarding the publication of this paper.

\section{References}

1 Maraqa NF, et al: Higher occurrence of hepatotoxicity and rash in patients treated with oxacillin, compared with those treated with nafcillin and other commonly used antimicrobials. Clin Infect Dis 2002;34:50-54.

-2 Viehman JA, et al: Adverse events lead to drug discontinuation more commonly among patients who receive nafcillin than among those who receive oxacillin. Antimicrob Agents Chemother 2016;60:30903095.

- Lee WM: Drug-induced acute liver failure. Clin Liver Dis 2013;17:575-586, viii.

-4 Pas AT, Quinn EL: Cholestatic hepatitis following the administration of sodium oxacillin. JAMA $1965 ; 191: 674-675$.

5 Lestico MR, Vick KE, Hetsko CM: Hepatic and renal dysfunction following nafcillin administration. Ann Pharmacother 1992;26:985-990.

6 Alam MB, Kadoura A, Sathaiah M: A fatal case of nafcillin-induced hepatotoxicity: a case report and the literature review. Case Rep Med 2012;2012:953714. 


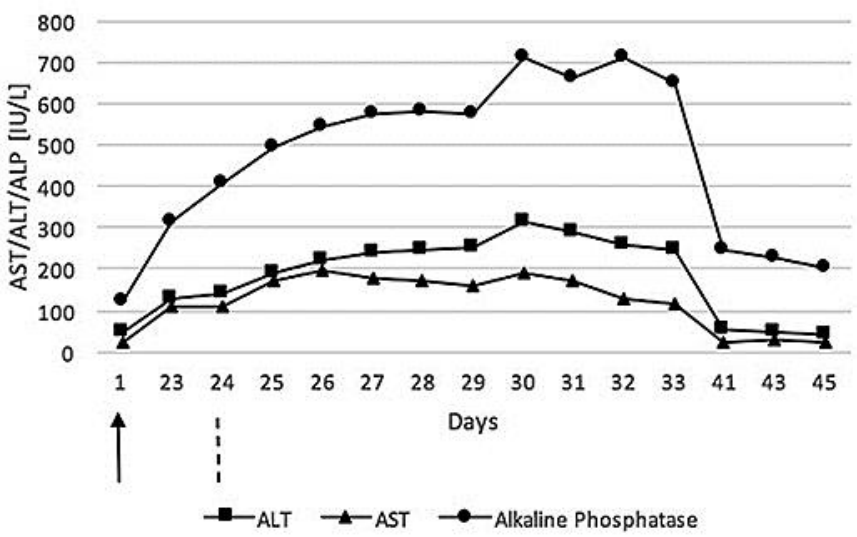

Fig. 1. Transaminase levels. Variation in AST, ALT, and ALP levels following initiation of nafcillin treatment. The solid arrow indicates initiation of antibiotic therapy (day 1) and the dashed line indicates withdrawal of treatment (day 24). AST, aspartate transaminase; ALT, alanine transaminase; ALP, alkaline phosphatase.

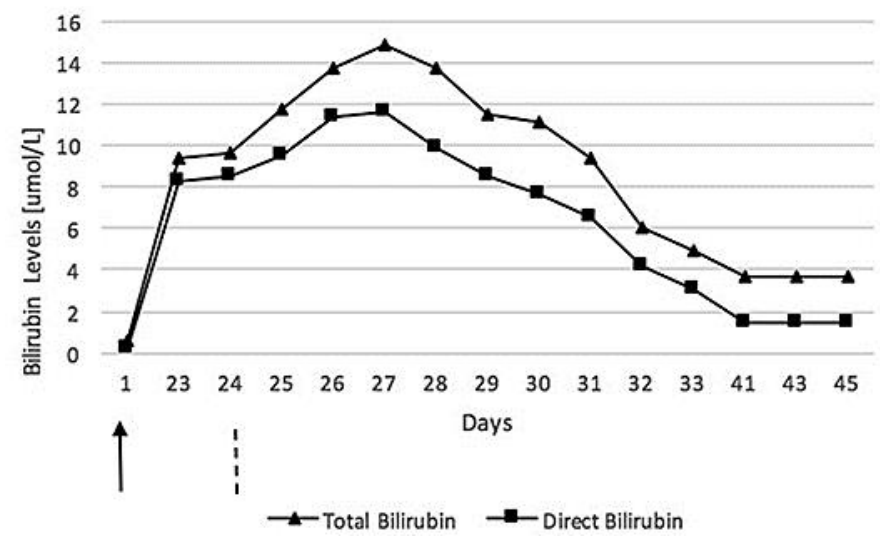

Fig. 2. Bilirubin levels. Variations in total and direct bilirubin levels following initiation of nafcillin treatment. The solid arrow indicates initiation of antibiotic therapy (day 1) and the dashed line indicates withdrawal of therapy (day 24). 